\title{
Descripción morfológica de los estadíos larvales I y II y redescripción de la hembra de Chaetanaphothrips orchidii Moulton (Thysanoptera: Thripidae), con comentarios sobre sus plantas hospederas
}

\author{
Morphological description of the larvae and redescription of \\ the female Chaetanaphothrips orchidii Moulton (Thysanoptera: \\ Thripidae), with comments on their host plants \\ D.0.I.: https://doi.org/10.30550/j.azl/2018.62.2/2
}

\author{
Ventura, Laura ${ }^{1-2^{*}}$; Silvia Tapia ${ }^{1-3}$; María I. Zamar ${ }^{4}$; Soledad Ochoa ${ }^{1}$; \\ Daniel Ortiz ${ }^{1}$ \\ 1 Estación Experimental de Cultivos Tropicales (EECT) INTA Yuto. Ruta Nacional 34 Kilómetro 1286 \\ (4518) Yuto, Jujuy (Argentina); ventura.laura@inta.gob.ar \\ 2 CONICET, Jujuy, Argentina. \\ 3 Facultad de Ciencias Agrarias, Universidad Nacional de Jujuy. Alberdi 35, (4600) San salvador de \\ Jujuy, Jujuy (Argentina). \\ 4 Instituto de Biología de la Altura, Universidad Nacional de Jujuy. Bolivia 1661, (4600) San Salvador \\ de Jujuy, Jujuy (Argentina).
}

- Resumen - Se describe por primera vez la morfología externa de los estadios larvales I y II, y se redescribe a la hembra de Chaetanaphothrips orchidii Moulton (Moulton, 1907), a partir de ejemplares obtenidos de la cría del insecto bajo condiciones de laboratorio y de muestras recolectadas sobre frutos de pomelo (Citrus paradisi Macfad) var. flame, foster y rouge; naranja (Citrus sinensis L.) var. valencia; limón (Citrus limon Burm) var. genova, y de la vegetación espontanea en floración provenientes de cultivos de cítricolas de las provincias de Jujuy y Salta (Argentina).

Palabras clave: Morfología, Citrus, vegetación espontánea, trips de la orquídea.

Abstract - The external morphology of larval stages I and II is described for the first time, and the female of Chaetanaphothrips orchidii Moulton is redescribed, from specimens obtained from the breeding of the insect under laboratory conditions and from samples collected on grapefruit fruits (Citrus paradisi Macfad) var. flame, foster and rouge; orange [Citrus sinensis L.) var. valencia; lemon (Citrus limon Burm) var. genova, and spontaneous flowering vegetation from citrus crops in the provinces of Jujuy and Salta (Argentina).

Keywords: Morphology, Citrus, spontaneous vegetation, orchid thrips.

\footnotetext{
> Ref. bibliográfica: Ventura, L.; Tapia, S.; Zamar M. I.; Ochoa S.; Ortiz, D. 2018. Descripción morfológica de los estadíos larvales I y II y redescripción de la hembra de Chaetanaphothrips orchidii Moulton (Thysanoptera: Thripidae), con comentarios sobre sus plantas hospederas. Acta zoológica lilloana 62 (2): 12-23.

> Recibido: 02/03/18 - Aceptado: $31 / 08 / 18$

$>$ URL de la revista: http://actazoologica.lillo.org.ar

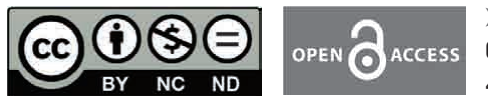

> Algunos derechos reservados. Esta obra está bajo una Licencia Creative Commons Atribución - No Comercial - Sin Obra Derivada 4.0 Internacional.
} 


\section{INTRODUCCIÓN}

El género Chaetanaphothrips contiene veinte especies probablemente originadas de la región Oriental, aunque algunas de ellas están ampliamente distribuidas (Masumoto, 2010). En América se encuentran Chaetanaphothrips orchidii (Moulton) Chaetanaphothrips signipennis (Bagnall) y Chaetanaphothrips leeuweni (Karny) (Mound y Marullo, 1996). Ch. orchidii se distingue claramente de las otras dos especies por presentar dos pares de setas posteroangulares, y de $C h$. leeuweni, por las dos bandas de color castaño de las alas anteriores.

Chaetanaphotrips orchidii se detectó en fincas citrícolas de Estados Unidos (Childers y Stansly, 2005; Childers y Nakahara, 2006), Panamá (Goldarazena, Gattesco, Atencio, Korytowski, 2012) y Brasil (Monteiro, Mound, Zucchi, 1999). En Argentina, Goane, Pereyra, Salas (2007) registraron por primera vez a esta especie en Tucumán donde ocasionó daños sobre frutos de limón; luego Tapia et al. (2014) la citaron sobre frutos de pomelo en las provincias de Salta y Jujuy.

Los daños ocasionados por Ch. orchidii se evidencian por la presencia de manchas bronceadas circulares o irregulares en el epicarpio como resultado de la alimentación y oviposición (Goane, et al., 2007). Se presentan en la zona de contacto entre frutos, o entre una rama $u$ hoja en roce directo con el fruto hasta la cosecha (Kono, 1984; Childers y Franz, 1994). Esas lesiones son superficiales, las que posteriormente originan una disminución en su valor comercial.

Actualmente la identificación taxonómica de $C h$. orchidii se realiza a partir de caracteres morfológicos externos de la hembra macróptera, debido a que se desconoce el macho (Mound y Marullo, 1996). Con respecto a las larvas, Stansly, Childers, Nigg, Simpson (2012) aportaron características generales referidas a la coloración del cuerpo y distinguieron la presencia de espinas diminutas en el noveno tergo abdominal. Cluever y Smith (2017), destacaron la forma de las setas y las espinas del tergo IX en una clave para la identificación de géneros de trips de interés agrícola.

La pérdida del valor comercial de los frutos cítricos por los daños de Ch. orchidii y las exigencias de inocuidad de los plaguicidas, determinan la necesidad de diferenciarlo del complejo de tisanópteros asociados a sistemas citrícolas basado en la detección temprana de la especie y su correcta identificación en cualquier estado de desarrollo. Por ello, los objetivos del presente trabajo son, describir las larvas I y II y redescribir la morfología externa de la hembra adulta de Ch. orchidii.

\section{MATERIALES Y MÉTODOS}

Los individuos de Ch. orchidii utilizados para realizar la descripción morfológica provinieron de dos fuentes. La primera, de la cría del tisanóptero sobre frutos de pomelo, y la segunda, de muestreos efectuados sobre frutos de Citrus paradisi var. flame, $C$. paradisi var. foster, $C$. paradisi var. rouge, $C$. sinensis var. valencia y $C$. limon var. genova y de la vegetación espontánea en floración provenientes de fincas citrícolas ubicadas en las localidades de Libertador General San

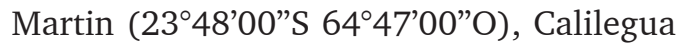
(2346'22"S 6446'11"O), de la provincia de Jujuy, y en Colonia Santa Rosa (232'00"S $\left.64^{\circ} 30^{\prime} 00^{\prime} O\right)$ de la provincia de Salta. La cría se efectuó en el insectario del Laboratorio de Zoología Agrícola y Forestal de la Estación Experimental de Cultivos Tropicales INTA Yuto. Se inició a partir de la emergencia de las primeras hembras obtenidas de huevos de Ch. orchidii en frutos de pomelo traídos del campo. Para obtener larvas y hembras se utilizaron jaulas de cría individuales con un fruto de pomelo limpio de $4 \mathrm{~cm}$ de diámetro, como substrato de alimentación y oviposición. Las jaulas se construyeron con recipientes plásticos transparentes de 250 $\mathrm{g}$, en cuya base se colocó papel absorbente. La abertura se cubrió con un trozo de film humedecido para evitar la deshidratación del fruto. Para simular el roce directo con los órganos de la planta se colocó en la parte superior del fruto un trozo circular de 
papel cartulina forrado en la cara superior con cinta adhesiva. En cada jaula se colocó una hembra para la oviposición. A los tres días, cada hembra fue trasladada a una jaula nueva y así sucesivamente hasta su muerte. Diariamente, se controló el nacimiento de las larvas I, las que eran extraídas con un pincel fino humedecido e inmediatamente acondicionadas en preparaciones microscópicas. Para obtener larvas II y adultos se procedió de la misma forma.

La descripción morfológica se realizó sobre la base de preparaciones microscópicas permanentes para adultos y semipermanentes para larvas siguiendo las técnicas de Mound y Kibby (1998). Las mismas quedaron depositadas en la coleccion del Laboratorio de Zoología Agrícola y Forestal de la Estación Experimental de Cultivos Tropicales (EECT) INTA Yuto y del Instituto de Biología de la Altura (INBIAL - UNJu).

a) Preparaciones microscópicas permanentes

$1^{\circ}$ : Maceración: se realizó en solución de $\mathrm{NaOH} 2 \%$ durante 24 a 72 horas; para facilitar la eliminación del contenido del cuerpo se punzó el abdomen con una aguja entomológica muy fina entre las coxas posteriores y se efectuó suaves presiones para estirar las antenas y patas.

$2^{\circ}$ : Lavado: en agua destilada dos o tres veces durante 5 '.

$3^{\circ}$ : Deshidratación: A través de pasajes sucesivos en alcohol a distintas concentraciones: 70\%: 30'; 80\%: 15'; 95\%: 10'; absoluto 100\%: 5 '.

4: Montaje: se colocó una gotita de bálsamo de Canadá en el centro del portaobjetos y otra en el cubreobjetos; para facilitar el extendido del trips se puso el espécimen en el cubreobjetos, con la parte ventral hacia arriba, extendiendo cuidadosamente las patas, antenas y alas; luego se invirtió el cubre y se ubicó sobre el portaobjetos presionando suavemente con una aguja entomológica.

Las preparaciones se llevaron a estufa a $35-40^{\circ} \mathrm{C}$ durante 1 o 2 semanas.

$5^{\circ}$ : Etiquetado: se dispuso la preparación con la cabeza del trips hacia abajo; a la derecha la etiqueta indica: planta hospedera, país, localidad, fecha de recolección, nombre del recolector y en la etiqueta de la izquierda se anotó nombre de la especie y autor, sexo, código de recolección.

b) Preparaciones microscópicas semipermanentes

Esta técnica se usó para facilitar las identificaciones de rutina y para larvas. Los individuos extraídos del líquido conservante, se lavaron 2 o 3 veces en agua destilada, luego se llevaron a alcohol al 70\% durante 24 horas y se montaron en Hoyer siguiendo los pasos de la técnica anterior.

Las medidas se expresaron en micras $(\mu)$, excepto el largo total (desde el borde cefálico al extremo del abdomen) y ancho máximo que se expresaron en milímetros ( $\mathrm{mm})$.

Para nombrar las estructuras morfológicas de la hembra se siguió la nomenclatura de Mound y Marullo (1996); mientras que para las larvas se aplicó la proporcionada por Heming (1991).

\section{RESULTADOS \\ Chaetanaphothrips orchidii \\ (Moulton), 1907}

Hembra macróptera (Figs. 1-11).- Color del cuerpo, segmentos antenales I, II, III, tres cuarto basal del VI, mitad basal del $\mathrm{V}$ al VI, patas y setas del cuerpo amarillo claro; cuarto distal del segmento antenal IV, la mitad distal del V al VI, línea longitudinal media de las alas posteriores y escamas de las alas anteriores castaño claro; base de las alas anteriores excepto dos bandas: subasal y apical, castaño oscuro; ovipositor amarillo; ocelos con la base anaranjado rojiza; ojos rojo oscuro (Fig. 1). Cabeza más ancha que larga, con las mejillas suavemente convergentes en la base, zona occipital con líneas de esculturas transversales con pocas bifurcaciones; con dos pares de setas ocelares, las del par I, ausentes; setas III dentro del triángulo ocelar; presenta generalmente tres pares de setas postoculares (i, ii y iii) (Fig. 2). Antenas de ocho segmentos, segmento III prolongado con pedicelo simple, segmento III y IV con conos sensoriales bifurcados, delgados y largos, dorsales y ventrales res- 


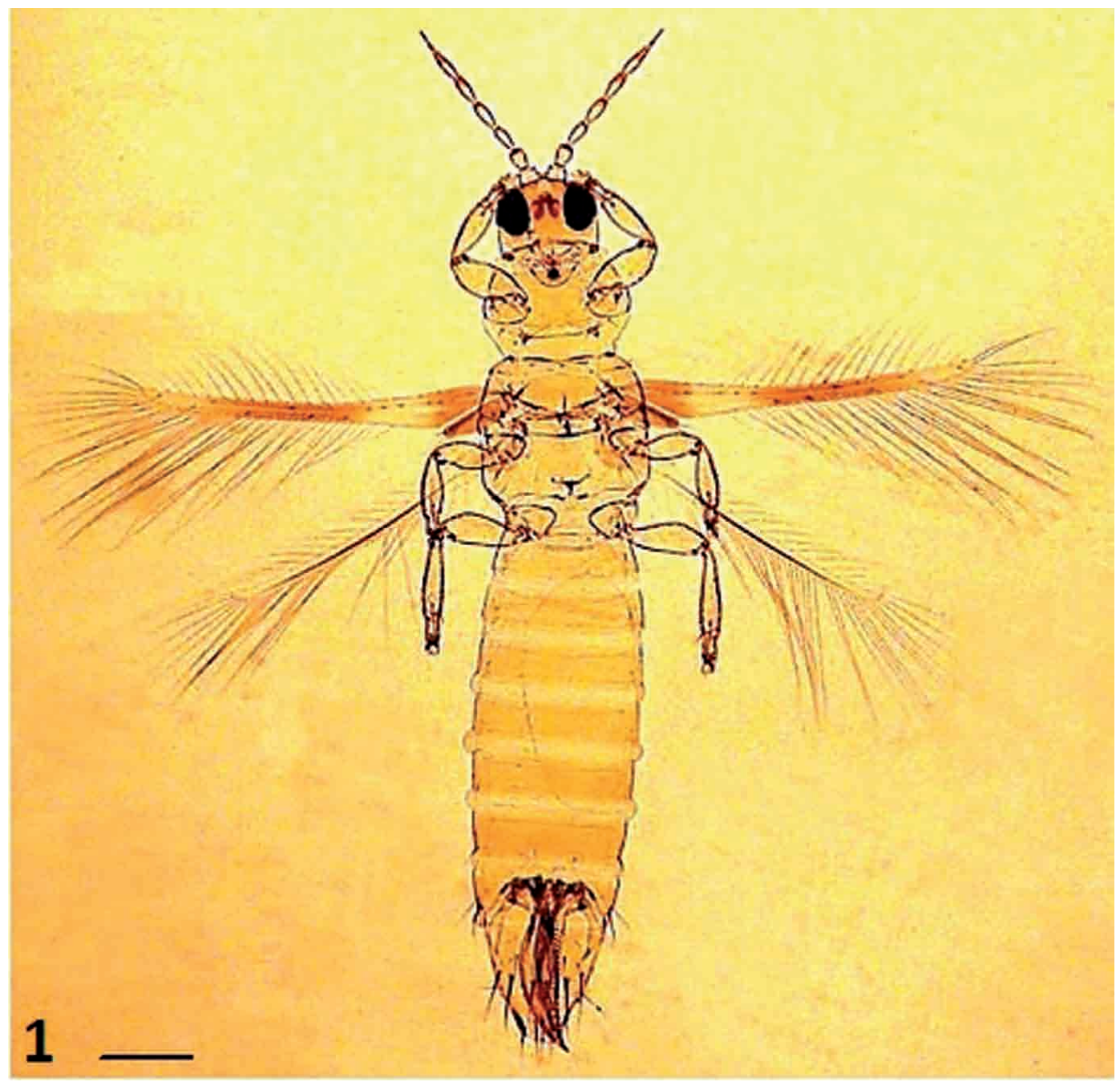

Fig. 1. Chaetanaphothrips orchidii Moulton, hembra. Habitus.

pectivamente (Figs. 3-4). Pronoto rectangular, superficie lisa excepto por unas escasas estrías próximas al margen anterior y posterior; setas anteromarginal, anteroangular, posteromarginal del mismo tamaño que las discales, estas últimas dispuestas en la mitad anterior del pronoto; setas posteroangulares internas más largas que las externas (Fig. 5). Mesonoto con 5-7 líneas esculturales bifurcadas principales que se anastomosan hacia los lados, las líneas del cuarto inferior se dirigen hacia la línea media, con un par de setas medias cortas. Metanoto débilmente reticulado en la zona media y hacia los lados con líneas verticales, con un par de setas medias anteriores ubicadas debajo del margen anterior y un par de setas medias próximas al margen posterior (Fig. 6). Alas anteriores con dos hileras incompletas de setas; primera vena con 8-10 setas, en la base presenta dos series de tres setas separadas por un intervalo y 3-4 setas separadas entre sí a lo largo de la vena; segunda vena con 3-4 setas; venas costal con 16 setas (Fig. 7). Furca mesotorácica con espina bien desarrollada. Tergos abdominales esculturados con estrías transversales más conspicuas en las zonas laterales, y con craspedum posteromarginal en forma de banda ancha (Fig. 8). Esternos abdominales con craspedum pos- 

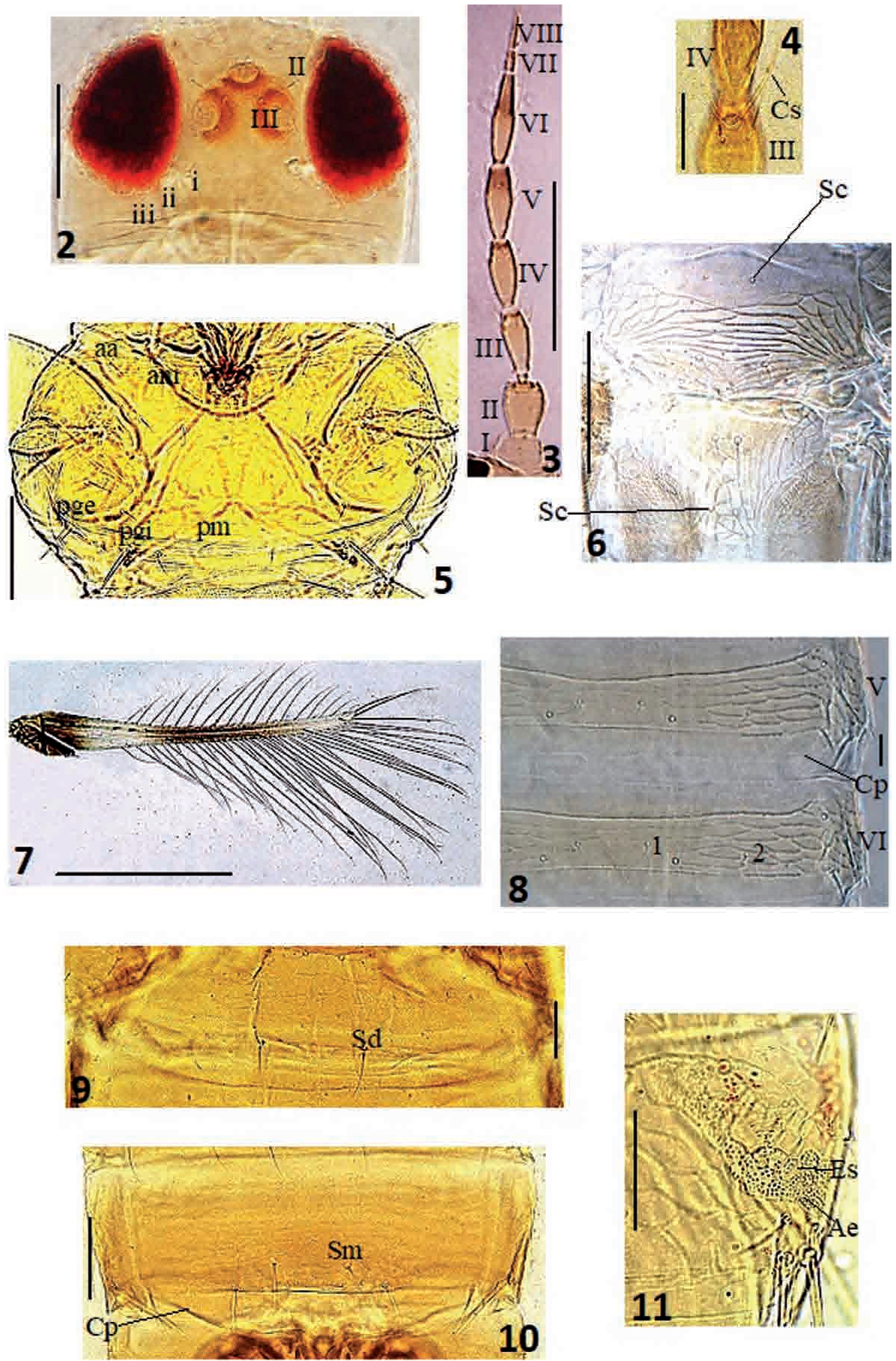

Figs. 2-11. Chaetanaphothrips orchidii Moulton, hembra. 2) Cabeza; 3) antena; 4) segmento antenal III-IV; 5) setas del pronoto; 6) mesonoto y metanoto; 7) primer par de alas; 8) tergos abdominales $\mathrm{V}-\mathrm{VI}$; 9) esterno abdominal II; 10) esterno abdominal VII; 11) tergo abdominal VIII. Referencias: Cs] cono sensorial, Sc) sensilia campaniforme, Sd] setas discales, Sm) setas medias, $\mathrm{Cp}$ ) craspedum posteromarginal, Es] espiráculo, Ae) área espiracular. Escalas: 1 y $3=0,1 \mathrm{~mm} ; 2=0,4 \mathrm{~mm} ; 4$ y $9=0,2 \mathrm{~mm} ; 5=0,5 \mathrm{~mm} ; 6=0,6 \mathrm{~mm} ; 7=0,3 \mathrm{~mm} ; 8$, 10 y $11=0,03 \mathrm{~mm}$. 
teromarginal lobulado excepto en la zona media del segmento VII (Fig. 10). Esterno abdominal II con una o un par de setas discales y sin área glandular transversal (Fig. 9), esterno abdominal VII con un par de setas marginales medias próximas al margen posterior (Fig. 10). Tergo abdominal VIII con un área granular o punteada alrededor del espiráculo (Fig. 11) y con peine incompleto de 12 microtriquias en la zona media. Setas S1 del tergo abdominal IX más cortas que las S2 y S3, setas S1 del segmento X más largas que las S2.

Medidas (cuerpo distendido).- Cuerpo, largo: $1 \mathrm{~mm}( \pm 0,1)$; ancho 0,2mm $( \pm 0,01)$. Cabeza, largo: 85 ( \pm 7$)$; ancho: $140( \pm 8)$. Setas ocelares II: $7( \pm 1)$, III: $12( \pm 2)$. Setas postoculares i: $7( \pm 2)$, postocular ii: $6( \pm 1)$. Segmentos antenales ancho, I: $26( \pm 2)$, II: 23 ( \pm 1$)$, III: $15( \pm 1)$, IV: 15( \pm 1$), \mathrm{V}: 16( \pm 2)$, VI: $13( \pm 1)$, VII: $5( \pm 1)$, VIII: $3( \pm 1)$; largo, I: $24( \pm 2)$, II: 33 ( \pm 3$)$, III: 49 ( \pm 2$),$ IV: 46 $( \pm 4), \mathrm{V}: 47( \pm 4), \mathrm{VI}: 54( \pm 3)$, VII: $12( \pm 1)$, VIII: 20 ( \pm 2$)$. Pronoto, largo: $95( \pm 5)$, ancho: 183 ( \pm 9$)$; setas am: $11( \pm 2)$; pg int.: $29( \pm 4)$; pg ext.: $22( \pm 3)$. Setas abdominales del segmento IX, S1: $85( \pm 5)$, S2: 94 ( \pm 6$),$ S3: 105 ( \pm 8$)$; setas del segmento $X$, S1: $84( \pm 4)$, S2: $70( \pm 6)$. Número de setas de la vena anterior del primer par de alas: 9 $( \pm 1)$, vena posterior: $4( \pm 0,3)$.

Material examinado.- ARGENTINA, JUJUY, Libertador General San Martin, 17-X-12, 1 o, ex/C. paradisi var. foster (frutos), col. Laura Ventura; 22-X-12, 2 우, ex/C. paradisi var. foster (frutos), col. Laura Ventura; 4-IV13, 1 ᄋ, ex/C. paradisi var. foster (frutos), col. Soledad Ochoa; 11-X-12, 2 우, ex/C. paradisi var. flame (frutos), col. Danielrtiz; 16-XI-12, 2 우, ex/C. paradisi var. flame (frutos), col. Silvia Tapia; 26-IV-13, 4 우, ex/B. pilosa (flores), col. Laura Ventura; 16$\mathrm{V}-13,3$ 우, ex/B. pilosa (flores), col. Laura Ventura; 28-I-13, 2 우, ex/B. pilosa (flores), col. Laura Ventura; 31-V-13, 4 우, ex/B. pilosa (flores), col. Laura Ventura; 7-VIII-13, 2 오, ex/Amaranthus hybridus L. (flores), col. LauraVentura; 19-IX-12, 1 \&, ex/A. hybridus (flores), col. LauraVentura; 14-II-13, 1 우, ex/A. hybridus (flores), col. LauraVentura; 16-V-13, 1 \&, ex/A. hybridus (flores), col. Laura Ventura; 14-II-13, 1 \&, ex/Commelina erecta (flores), col. Laura Ventura; 4-IV-13, 2 오, ex/Justicia goudotii (flores), col. Laura Ventura; 4-IV-13, 1 \%, ex/Malvastrum coromandelianum (flores) col. Laura Ventura; 31-V-13, 4 우, ex/Bidens pilosa spp. pilosa (flores), col. Laura Ventura; 14-II-13, 1 오, $\mathrm{ex} / M$. coromandelianum (flores), col. Laura Ventura; 4-IV-13, 4-IV-13, 1 오, ex/Cestrum parqui LHér. (hojas), col. Laura Ventura; Calilegua, 10-X-12, 1 ㅇ, ex/C. sinensis var. valencia (frutos), col. Soledad Ochoa; 16$\mathrm{XI}-12,1$ Q, $\mathrm{ex} / C$. sinensis var. valencia (frutos), col. Daniel Ortiz; 4-IV-13, 1 ᄋ, ex/Sida rhombifolia L. (flores), col. Laura Ventura; 31-V-13, 2 우, ex/Thunbergia alata Bojer ex Sims (flores), col. Laura Ventura; 18-IX-12, 2 우, ex/C. limón var. genova (frutos), col. Soledad Ochoa; 31-V-13, 1 o, ex/S. rhombifolia (flores), col. Laura Ventura; Yuto, 25-IV13, 2 우, ex/C. paradisi var. foster (frutos), cría de laboratorio (INTA EECT), col. Laura Ventura; 19-IV-13, 2 오, ex/C. paradisi var. rouge (frutos), cría de laboratorio (INTA EECT), col. Laura Ventura; 11-V-13, 2 우, ex/C. paradisi var. rouge (frutos), cría de laboratorio (INTA EECT), col. Laura Ventura. SALTA, Col. Sta. Rosa, 10-X-12, 1 \&, ex/C. paradisi var. rouge (frutos), col. Soledad Ochoa; 28-XII-12, 1 ㅇ, ex/C. paradisi var. rouge (frutos), col. Daniel Ortiz; 19-IV-13, 2 우, ex/C. paradisi var. rouge, col. Laura Ventura; 6-IX-12, 1 Q , ex/C. parqui (hojas), col. Silvia Tapia; 24-V-13, 1 ᄋ, ex/M. coromandelianum (flores), col. Laura Ventura; 24-V-13, 3 우, ex/A. hybridus (flores), col. Laura Ventura.

Descripción de la larva I (Figs. 12-15).Cuerpo de color blanquecino y al finalizar el estadio cambia a amarillo pálido, ojos rojizos, último segmento antenal castaño, los restantes antenitos y patas, blanquecinas. Setas dorsales con los ápices flecados y agudos, setas esternales agudas. Cuerpo con placas dorsales oblongas pequeñas portadoras de microtriquias distales, menos numerosas en 
la cabeza y zona central del pronoto, poco notorias en la superficie ventral. Las placas de los tergos y esternos abdominales se disponen en hileras transversales de 4-5 y de
5-6 respectivamente; tergo IX con estrías entrecortadas de microtriquias diminutas en la zona media anterior del segmento, más desarrolladas sobre las setas 1 y 2 ; peine
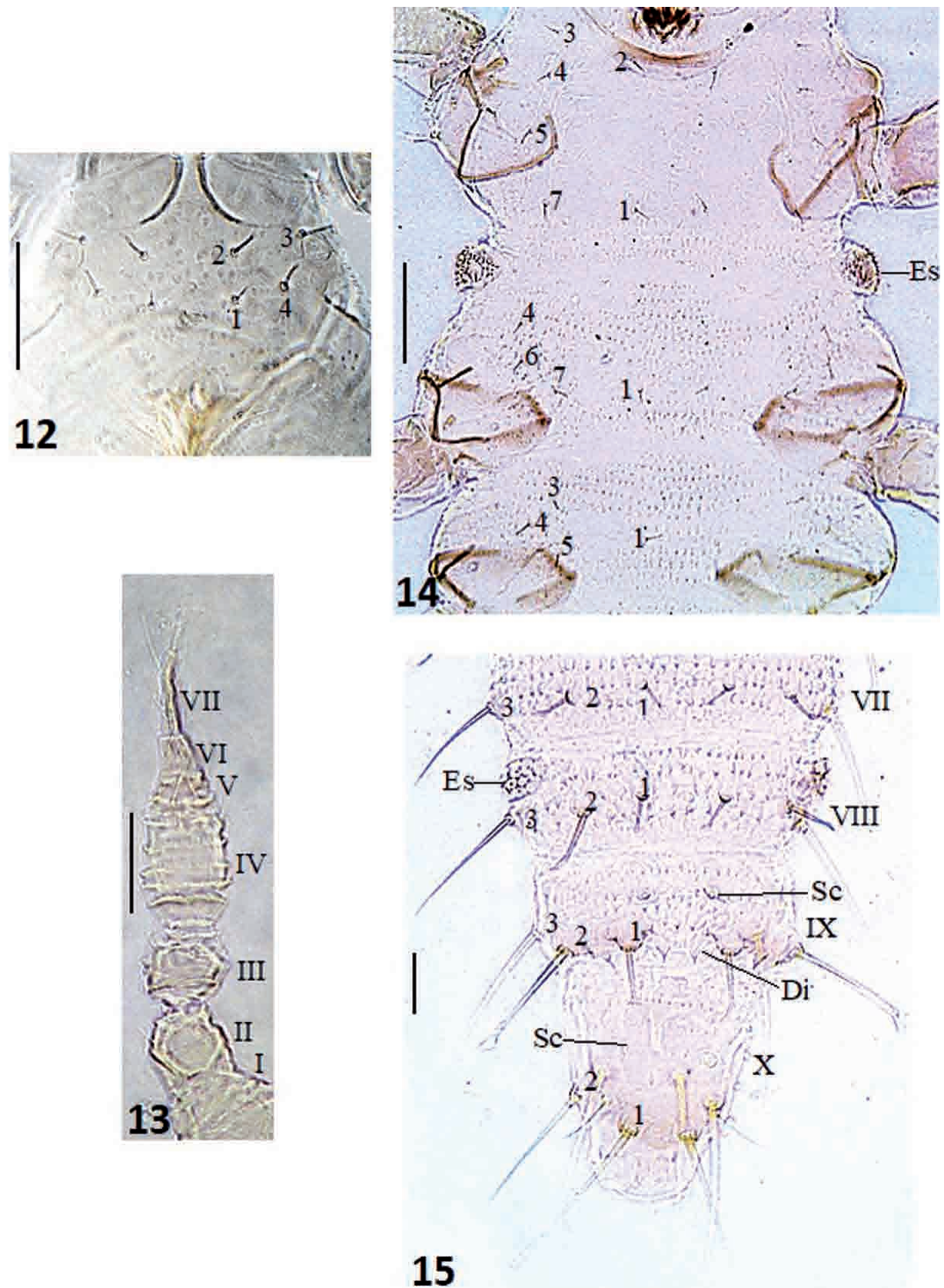

Figs. 12-15. Chaetanaphothrips orchidii Moulton, larva I. 12, cabeza; 13, antena; 14, pronoto, mesonoto y metanoto; 15, tergos abdominales VII-X. Referencias: Es - espiráculo, Sc - sensilia campaniforme, Di - diente. Escalas: 12 y $13=0,3 \mathrm{~mm} ; 14=0,03 \mathrm{~mm} ; 15=$ $0,1 \mathrm{~mm}$. 
generalmente con 2-3 dientes medios entre las setas 1 . Cabeza más ancha que larga, con cuatro pares de setas cefálicas muy delgadas y ápices flecados, el par de setas 5, está ausente (Fig. 12). Antenas de siete segmentos, segmento I con una seta lateral interna curva; segmento II con estrías sin microtriquias, con un poro sensorial entre las bases de las setas distales y una seta media ventral apical larga; segmento III pedicelado en forma de copa con cuatro estrías, sin microtriquias y dos setas sensoriales medias; segmento IV con cinco estrías de escasas microtriquias , dos pares de setas sensoriales medias y una lateral externa; segmento $\mathrm{V}$ con un cono dorsal y una seta lateral interna larga curva; segmento VI con un cono lateral; segmento VII con cuatro setas laterales y una distal (Fig. 13). Pronoto con seis pares de setas, el par seis, ausente; mesonoto con cuatro pares de setas, faltan los pares 2,3 y 5 , y el metanoto presenta cuatro pares de setas, falta el par 2 (Fig. 14). Segmentos abdominales I, IX y X con dos pares de setas dorsales, II-VIII con tres pares; segmento IX con dos sensilias campaniformes sobre la segunda hilera de microtriquias, las setas 1 y 2 de este segmento con los ápices flecados y las setas 3 , agudas, muy pequeñas; también presenta generalmente tres espinas entre las setas 1 ; segmento $\mathrm{X}$ con el par de setas 1 de ápices flecados y setas 2 agudas, y dos sensilias campaniformes (Fig. 15). Espiráculos en el mesotórax y segmentos abdominales II y VIII (Figs. 14, 15).

Medidas (cuerpo distendido).- Cuerpo, largo: $0,5 \mathrm{~mm}( \pm 0,1)$, ancho: $0,1 \mathrm{~mm}$ $( \pm 0,02)$. Cabeza, largo: $57( \pm 4)$, ancho: 92 $( \pm 5)$. Setas cefálicas, 1: $3( \pm 1), 2: 5( \pm 1)$, 3: $7( \pm 1), 4: 5( \pm 1)$. Segmentos antenales largo, I: 11 ( \pm 2$)$, II: 20 ( \pm 2$)$, III: $24( \pm 4)$, IV: $37( \pm 3)$, V: $8( \pm 2)$, VI: $11( \pm 1)$, VII: 21 ( \pm 3$)$; ancho, I: 26 ( \pm 3$)$, II: 22 ( \pm 2$)$, III: 24 ( \pm 2$), \mathrm{IV}: 25( \pm 2), \mathrm{V}: 15( \pm 1)$, VI: $9( \pm 1)$, VII: $4( \pm 1)$; Setas pronotales, 1: $5( \pm 1), 2$ : $5( \pm 1), 3: 4( \pm 1), 4: 4( \pm 2), 5: 5( \pm 1), 7$ : $5( \pm 1)$. Espinas, largo: $3( \pm 0,4)$, ancho: 3 $( \pm 0,5)$. Número de espinas entre las setas 1 del segmento abdominal IX: $3( \pm 1)$.
Material examinado. - ARGENTINA, JUJUY, Yuto, 26-IV-13, L I: 5, ex/Citrus paradisi var. flame (frutos), cría de laboratorio (INTA EECT), col. Luara Ventura; 9-V-13, L I: 4, ex/ C. paradisi var. flame (frutos), cría de laboratorio (INTA EECT), col. Laura Ventura; 26-IV13, L I: 5, ex/C. paradisi var. foster (frutos), cría de laboratorio (INTA EECT), col. Laura Ventura. SALTA, Colonia Santa Rosa, 24-I-14, L I: 1 , ex/C. paradisi var. rouge (frutos), col. Laura Ventura.

Descripción de la larva II (Figs. 16-21).Cuerpo de color amarillo, al finalizar el estadio cambia a rosáceo; primer segmento antenal del mismo color del cuerpo, el resto castaño claro. Placas cefálicas y protorácicas redondeadas. Placas mesotorácicas y metatorácicas oblongas, con microtriquias distales y con setas flecadas de bases elevadas. Esternos de tórax y abdomen con placas ventrales oblongas más pequeñas que las dorsales. Cabeza más ancha que larga, con cinco pares de setas cefálicas: setas 1, 2 y 3 con ápices flecados; setas 4 con ápices agudos; setas 5 de ápices romos (Fig. 16). Antenas de siete segmentos, segmento I con una seta lateral interna curvada; segmento II con un poro sensorial entre las bases de las dos setas distales; segmento III pedicelado, alargado con tres estrías sin microtriquias y con dos setas medias distales, segmento IV pedicelado con cinco hileras de microtriquias, con un cono sensorial ventral y dos setas laterales; segmento $\mathrm{V}$ corto rectangular con un cono sensorial externo más largo que el segmento IV; segmento VI con cono sensorial externo bien desarrollado llegando a la mitad del segmento VII con dos setas laterales; segmento VII alargado, dorsalmente con tres setas laterales y una distal aguda; segmento I, II, VI y VII sin estrías ni microtriquias (Fig. 17). Protórax trapezoidal, con las setas $1 \mathrm{y}$ 3 agudas; setas 2, 4, 5, 6 y 7 flecadas; setas 6 bien desarrolladas, aproximadamente 2/3 más largas que las setas 1 . Mesotórax rectangular, con las setas 1, 2, 4, 5, 6 y 7 flecadas, setas 2 agudas y un par de setas auxiliares (Fig. 18). Metatórax con cinco pares de setas flecadas (Fig. 19). Tergos abdominales I 
y X con dos pares de setas, un par de setas auxiliares sobre las setas 2 del segmento I; II a VIII con tres pares de setas (Fig. 20); el VIII sin sensilias campaniformes; segmento IX con dos sensilias campaniformes sobre la segunda hilera de microtriquias, las setas 1 y 2 de este segmento con los ápices flecados y las setas 3 , agudas, muy pequeñas; también presenta generalmente cuatro espinas entre las setas 1; segmento X con el par de
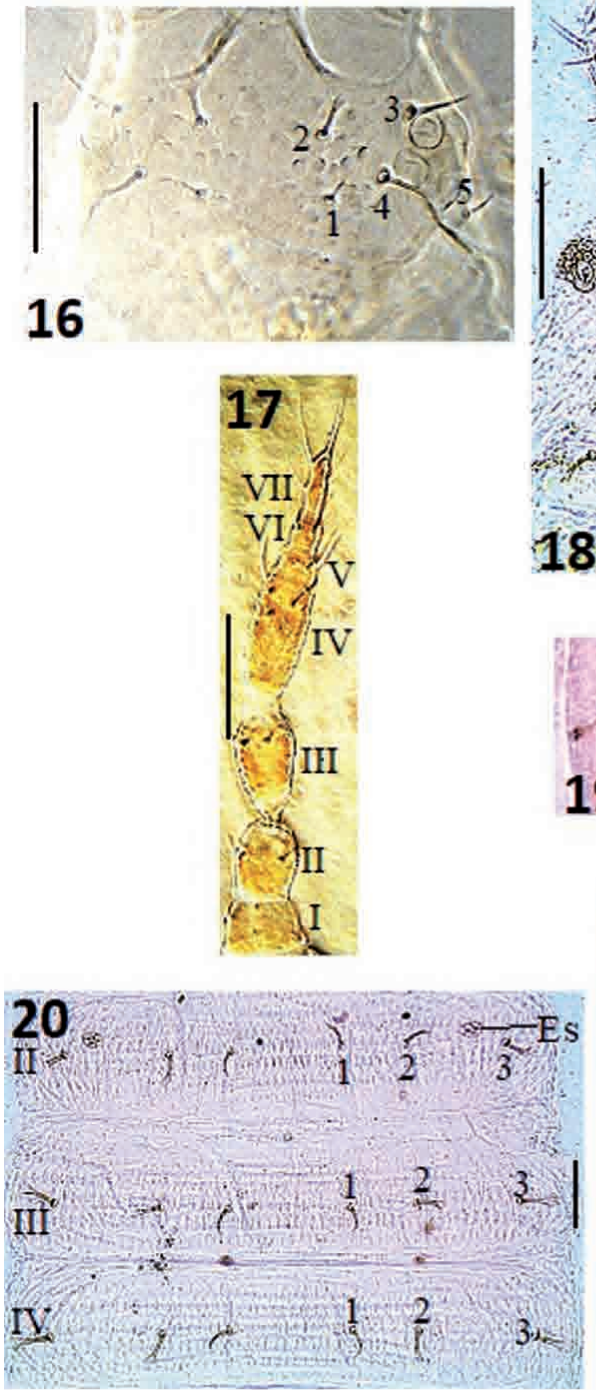
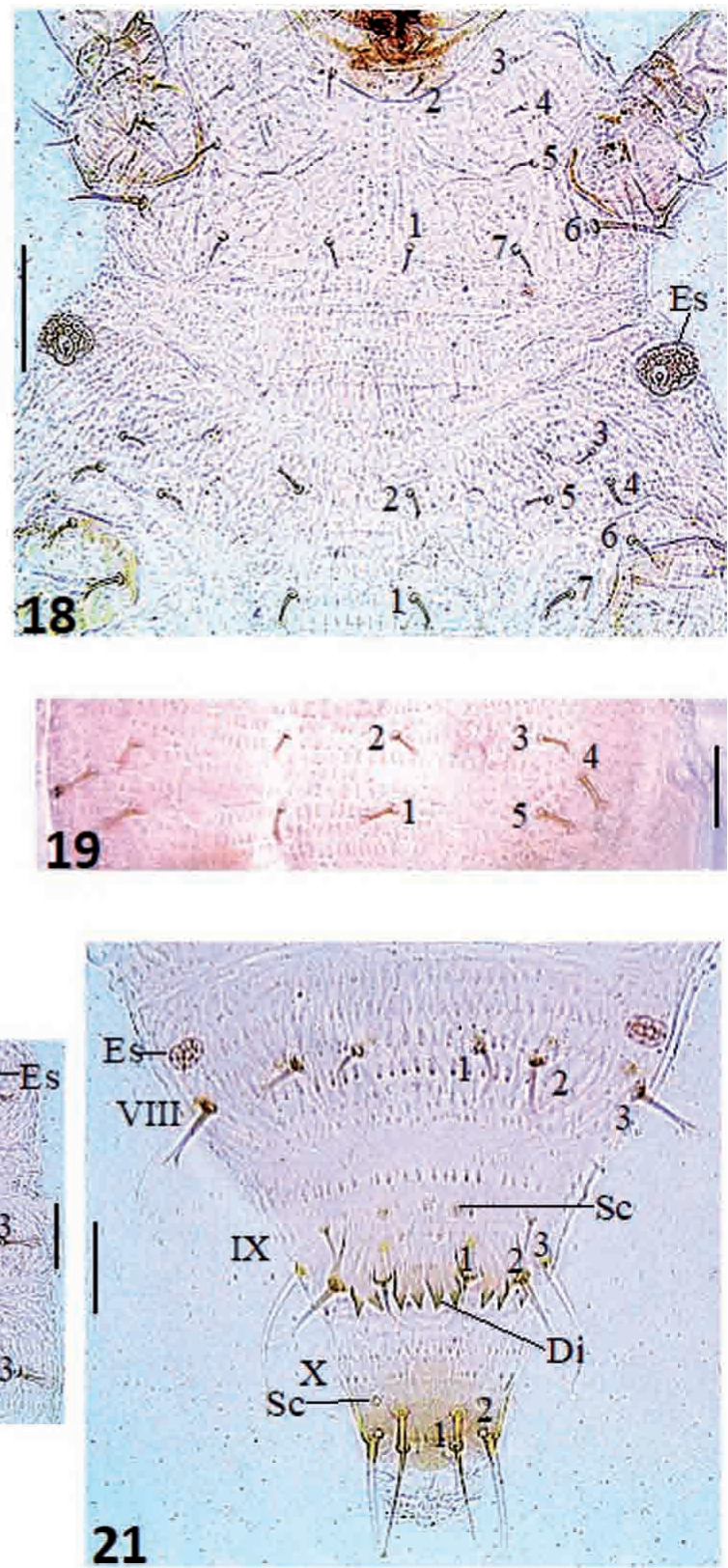

Figs. 16-21. Chaetanaphothrips orchidii Moulton, larva II. 16, cabeza; 17, antena; 18, pronoto y mesonoto; 19, metanoto; 20, tergos abdominales IIIV; 21 , tergos abdominales VIII-X. Referencias: Es - espiráculo, Sc - sensilia campaniforme, Di - diente. Escalas: 16 y $17=$ $0,04 \mathrm{~mm} ; 18,19$ y $21=0,03 \mathrm{~mm} ; 20=0,3 \mathrm{~mm}$. 
setas 1 de ápices flecados y setas 2 agudas, y dos sensilias campaniformes(Fig. 21). Espiráculos presentes en el mesotórax y en los segmentos abdominales II y VIII (Figs.18, 20 y 21).

Medidas (cuerpo distendido).- Cuerpo, largo: $1 \mathrm{~mm}( \pm 0,1)$, ancho: $0,3 \mathrm{~mm}( \pm 0,02)$. Cabeza, largo: $74( \pm 3)$, ancho: $96( \pm 8)$. Setas cefálicas, 1: $6( \pm 1), 2: 11( \pm 1), 3: 15$ $( \pm 1), 4: 10( \pm 2), 5: 8( \pm 1)$. Segmentos antenales ancho, I: $25( \pm 3)$, II: $19( \pm 2)$, III: 19 ( \pm 3$),$ IV: $17( \pm 3), \mathrm{V}: 13( \pm 3), \mathrm{VI}: 10( \pm 1)$, VII: $6( \pm 1)$; largo, I: 15 ( \pm 2$)$, II: $24( \pm 4)$, III: 37 ( \pm 7$)$, IV: $42( \pm 3)$, V: $8( \pm 2)$, VI: 12 ( \pm 3$)$, VII: $23( \pm 2)$. Setas pronotales, 1: 11 $( \pm 1), 2: 9( \pm 1), 3: 7( \pm 1), 4: 9( \pm 1), 5$ : $11( \pm 2), 6: 30( \pm 3), 7: 11( \pm 2)$. Espinas, largo: $3( \pm 1)$, ancho: $3( \pm 1)$. Numero de espinas entre las setas 1 del segmento abdominal IX: $4( \pm 1)$.

Material examinado.- ARGENTINA, JUJUY. Libertador General San Martin, 22-XI12, L II: 3, ex/C. paradisi var. foster (frutos), col. Laura Ventura; 17-X-12, L II: 3, ex/C. paradisi var. flame (frutos), col. Soledad Ochoa; 11-X-12, L II: 3, ex/C. paradisi var. flame (frutos), col. Daniel Ortiz; 31-V-13, L II: 2, ex/Bidens pilosa L. (flores). Yuto, 10-V13, L II: 9, ex/C. paradisi var. rouge (frutos), cría de laboratorio (INTA EECT), col. Laura Ventura; 24-I-14, L II: 3, ex/C. paradisi var. rouge (frutos), cría de laboratorio (INTA EECT), col. Laura Ventura; 9-V-13, L II: 3, ex/C. paradisi var. foster (frutos), cría de laboratorio (INTA EECT), col. Laura Ventura; 11-II-14, L II: 3, ex/C. paradisi var. foster (frutos), cría de laboratorio (INTA EECT), col. Laura Ventura. SALTA, Colonia Santa Rosa, 17-XI-13, L II: 5, ex/C. paradisi var. rouge (frutos), col. Laura Ventura.

\section{DISCUSIÓN}

Vierbergen et al. (2010) señalan que las larvas II de Dendrothripinae, Sericothripinae y parte de Thripinae carecen de sensilias campaniformes en el tergo VIII como se observa en la larva II de Chaetanaphothrips.
Vance (1974) destaca que la larva de Chaetanaphothrips presenta setas expandidas similares a las de Sericothrips. Al presente no existen descripciones detalladas de las larvas de otras especies de Chaetanaphothrips que permitan establecer comparaciones con la información aportada en este estudio.

Con respecto a las plantas hospederas, Hara et al. (2002), destacan que Ch. orchidii es polífaga, aunque tiene cierta preferencia por el Anthurium sp. También fue citada sobre ornamentales, como Dendrobium sp. Sw, Begonia sp. L., Strelitzia sp. Ait., Chrysanthemum sp. L., cultivos de cítricos, banano, batata, y en la vegetación adventicia fue recolectada sobre Tradescantia fluminensis Vell., Commelina erecta L., poáceas como Coix lacryma-jobi L.; Digitaria pruriens Fisch. ex Trin., Panicum purpurascens Raddi, Paspalum conjugatum (P.J.Bergius), Paspalum orbiculare G.Forst. P., Digitaria insularis (L.) Mez ex Ekman, Bidens alba (D.C.) L., Glechoma hederacea L., Fumaria officinalis L., Chenopodium ambrosioides L. y Rhynchelytrum repens (Willd.) C.E. Hubb. (Sakimura, 1975; Hara et al., 2002; Childers y Nakahara, 2006; Goane, et al., 2007).

En este estudio se registró la presencia de adultos de $C h$. orchidii en la vegetación espontánea que se muestreó en los cultivos citrícolas; en flores de Bidens pilosa L. (única planta en donde también se hallaron larvas), Justicia goudotii V.A.W. Graha, Thunbergia alata Bojer ex Sims, Malvastrum coromandelianum (L.) Garcke, Sida rhombifolia L., Amaranthus hybridus L. y en hojas de Cestrum parqui L'Heritier.

\section{AGRADECIMIENTOS}

A los técnicos y operarios de la Empresa Ledesma S. A. por permitirnos realizar los muestreos en las fincas de Libertador General San Martín, Calilegua y Colonia Santa Rosa. A la Dra. G. Entrocassi de la Cátedra de Botánica Sistemática de la Facultad de Ciencias Agrarias por ayudarnos con la identificación de las especies botánicas. A los miembros del área de Fitopatología de la EECT INTA Yuto, Palinología de la Facultad de Ciencias 
Agrarias de la Universidad Nacional de Jujuy (UNJu), y Entomología del Instituto de Biología de la Altura por colaborar con el empleo de los equipos de microscopia.

\section{FINANCIAMIENTO}

Este estudio ha sido financiado mediante los proyecto de investigación «Tisanópteros asociados a sistemas citrícolas de Jujuy y Salta» y «Bases para la implementación del manejo integrado de Chaetanaphothrips orchidii (Insecta: Thysanoptera) en plantaciones de pomelo de Jujuy y Salta», Res. C.S. No 00168-11 de la Secretaría de Ciencia y Técnica y Estudios Regionales de la Universidad Nacional de Jujuy, de la Beca CIN Res. P No 230/13, 364/14 y dentro del marco de Proyectos Regionales con Enfoque Territorial del INTA Pedemonte y las Yungas Código SALJU- 1232206.

\section{PARTICIPACIÓN}

En el presente trabajo la primera autora contribuyó con los muestreos, acondicionamiento, cría y caracterización morfológica del material de estudio y redacción del manuscrito. La segunda autora participó en las tareas de logística, contacto con los productores, muestreos y en la escritura del manuscrito. La tercera autora participó con la caracterización morfológica del material de estudio, tareas de logística y también colaboró con la escritura. Los cuartos y quintos autores participaron en los muestreos y acondicionamiento del material de estudio en el laboratorio.

\section{CONFLICTOS DE INTERÉS}

En el presente trabajo no existen conflictos de interés entre los autores o con terceros.

\section{LITERATURA CITADA}

Childers, C., Franz, G. (1994). Ring spot damage to Florida citrus fruit caused by trips feeding injury. Citrus Industry, 75, 38-43.

Childers, C., Stansly, A. (2005). Thrips (Thysanoptera: Thripidae) pest of Florida grapefruit: biology, seasonal and relative abundance, fruit damage and monitoring. Proceedings of the Florida State Horticultural Society, 118, 54-61.

Childers, C., Nakahara, S. [2006]. Thysanoptera (thrips) within citrus orchards in Florida: Species distribution, relative and seasonal abundance within trees, and species on vines and ground cover plants. Journal of Insect Science, 6, 1-19.

Cluever, J., Smith, H. (2017). A Photo-Based Key of Thrips (Thysanoptera) Associated with Horticultural Crops in Florida. Florida Entomologist, 100, 454-467.

Goane, L., Pereyra, V., Salas, H. (2007). Presencia de Chaetanaphothrips orchidii (Insecta: Thysanoptera: Thripidae) en fincas de limonero en Tucumán, Argentina. Revista Industrial y Agrícola de Tucumán, 84, 25-27.

Goldarazena, A., Gattesco, F., Atencio, R., Korytowski, C. (2012). An updated checklist of the Thysanoptera of Panama with comments on host associations. Check List, 8, 1232-1247.

Hara, A. H., Jacobsen, C., DuPonte, R. (2002). Anthurium Thrips Damage to Ornamentals in Hawaii. https://www. ctahr. hawaii.edu/oc/freepubs/pdf/IP9.pdf (Accedido: diciembre de 2017).

Heming, B. S. 1991. Order Thysanoptera. In: Stehr, F. H. (ed.). Immature Insects, Kendall Hunt, Dubuque, lowa, vol. 2, pp. $1-21$.

Kono, T. (1984). California Plant Pest and Disease Report. Volume 3 Number 3. In Plant Pest Diagnostics Center Publications. California Dept. of Food and Agriculture. https://www.cdfa.ca.gov/ plant/ppd/publications/cppdr.html (Accedido: diciembre de 2017).

Masumoto, M. (2010). Key to Genera of the Subfamily Thripinae (Thysanoptera: Thripidae) Associated with Japanese Plant Quarantine. Research Bulletin of The Plant Protection Service Japan, 46, 25-59.

Monteiro, R., Mound, L., Zucchi, R. (1999). Trips (Tysanoptera) as pest of plant production in Brazil. Revista Brasileira de Entomologia, 43, 163-171.

Moulton, D. (1907). A contribution to our knowledge of the Thysanoptera of California. Technical series, US Department of Agriculture, Bureau of Entomology, Technical Series, 12, 39-68.

Mound, L., Marullo, R. (1996). The trips of Central and South America: an introduction (Insecta: Thysanoptera). Gainesville, Florida: Memoirs on Entomology Inter- 
national.

Mound, L., Kibby, G. (1998). Thysanoptera. An Identification Guide. 2nd ed. Wallingford: CAB International.

Stansly, P., Childers, C., Nigg, H., Simpson, S. [2012]. Florida Citrus Pest Management Guide: Plant Bugs, Chewing Insect Pests, Caribbean Fruit Fly, and Thrips. ENY-605. URL: http://edis.ifas.ufl. edu/pdffiles/CG/CG00500.pdf (Acceso: diciembre de 2017].

Sakimura, K. (1975). Danothrips trifasciatus, new species, and collections notes on the Hawaniian species Danothrips and Chetanaphothrips (Thysanoptera: Thripidae). Proceedings, Hawaiian Entomologi- cal Society, 22, 125-132.

Tapia, S., Zamar M. I., Ventura L., Ochoa S., Ortiz D. (2014). Tisanópteros asociados a plantaciones citrícolas de Jujuy y Salta. Revista Agronómica del Noroeste Argentino. 34 (2): 251-253.

Vance, T. (1974). Larvae of the Sericothripini [Thysanoptera: Thripidae], with reference to other larvae of the Terebrantia, of Illinois. Illinois Natural History Survey Bulletin, 32, 145-208.

Vierbergen, G., Kucharczyk H., Kirk W. (2010) A key to the second instar larvae of the Thripidae of the Western Palaearctic región (Thysanoptera). Tijdschrift voor Entomologie 153: 99-160. 